Adv. Math. Econ. 2, 41-66 (2000)

@Springer-Verlag 2000

\title{
Job matching: a multi-principal, multi-agent model*
}

\author{
Tatsuro Ichiishi ${ }^{1}$ and Semih Koray ${ }^{2}$ \\ 1 Department of Economics, Ohio State University, 1945 North High Street, \\ Columbus, OH 43210-1172, USA \\ (e-mail: ichiishi@economics.sbs.ohio-state.edu) \\ 2 Department of Economics, Faculty of Economics, Administrative and Social Sci- \\ ences, Bilkent University, 06533 Bilkent, Ankara, Turkey \\ (e-mail: ksemih@bilkent.edu.tr)
}

Received: September 7, 1999

Revised: September 27, 1999

JEL classification: D82

Mathematics Subject Classification (1991): 90A35, 90A36

Abstract. A version of Spence's "job market" model is constructed and studied: There are two groups of individuals, the job applicants (the informed), and the incumbents (the uninformed). The applicants have private information about their types, and their actions (choice of education levels) serve as messages. The incumbents (employers) have only one type, and are endowed with differentiated information structures on actions of the informed. A contract is a pair of an education level and a wage level, and a wage schedule specifies a contract for each education level. The incumbent set is finite, and is fixed throughout the analysis (so free entry/exit is excluded). The paper studies endogenous determination of the wage schedules offered by the incumbents. The applicants behave noncooperatively. Two equilibrium concepts are proposed: a noncooperative equilibrium, a version of the Nash equilibrium which postulates noncooperative and passive behavior of the incumbents, and a cooperative equilibrium, a version of the strong equilibrium which postulates cooperative and passive behavior of the incumbents. It is shown that a cooperative equilibrium does not exist. By studying noncooperative equilibria, which do exist in many cases, it is concluded that it is not the informational advantage (defined as the abundance of measurable sets), but rather possession of the right information (in the sense that it best serves the needs of applicants) that enables an incumbent to win.

Key words: job matching, multi-principal multi-agent model, hidden information, hidden action, signalling

* The research reported in the present paper started while Tatsuro Ichiishi was visiting İktisat Bölümü (Department of Economics), Bilkent Üniversitesi in the Fall Semester, 1997. The authors would like to thank Murat Sertel and an anonymous referee for suggestions and comments, and Bilkent University for making this collaborative work possible. Tatsuro Ichiishi would also like to thank the people of Bilkent for their warm hospitality throughout his stay in Ankara. 


\section{Introduction}

The role of asymmetric information in allocation of resources, together with the associated information-revelation process, has long been a central focus of economic research. While the bulk of the literature addresses these issues within the framework of principal-agent relationship, which essentially reduces the problem to the sole principal's (the sole Stackelberg leader's) optimization problem subject to the agents' (the Stackelberg followers') responses, there are recent attempts to extend analysis to other economic setups characterized by different relationships among decision-makers.

A notable strand of such attempts is the core analysis of incomplete information. Here, there is no Stackelberg-type relationship, and more importantly the players can talk to each other for coordinated choice of strategies. See, e.g., Wilson (1978) for a pioneering work; Yannelis (1991) for formulation of feasibility of a strategy as its measurability; Ichiishi and Idzik (1996) for introduction of Bayesian incentive-compatibility to this strand; Ichiishi, Idzik and Zhao (1994) for information revelation (that is, endogenous determination of updated information structures); Ichiishi and Radner (1997) and Ichiishi and Sertel (1998) for studies of a specific model of Chandler's firm in multidivisional form for sharper results; and Vohra (1999) for a recent work. It is a common postulate in these works that every player takes part in design of a mechanism and also in execution of the signed contract.

The present paper provides an analysis of the role of asymmetric information, given yet another player relationship: We retain the principal-agent relationship, but allow for several principals in addition to several agents. Interaction of the principals is a focus of the paper. It is true that the traditional principal-agent literature frequently postulates existence of many principals, in fact infinitely many potential principals as required by the pure competition assumption (specifically, by the free entry and exit assumption), but this assumption in a nutshell reduces the model to the one-principal case in which the principal's only economically feasible strategy is the competitive strategy; this point was emphasized in Ichiishi (1997, Sections 7.4 and 7.6).

Given a multi-principal, multi-agent setup, we intend to study the roles of incomplete information about exogenous data and of incomplete information about endogenous variables. A general theory is yet to be developed, and our work reported in this paper is modest: As the first step towards a healthy general theory, we construct and study a very specific model, a variant of Spence's (1974) education model. There are two groups of individuals, the job applicants (the informed), and the incumbents (the uninformed). The applicants have private information about their types (productivity levels), and their actions (choice of education levels) serve as messages to the incumbents. The incumbents (employers) have only one type, have no information about applicants' types, only partially observe applicants' actions, and their strategies are to determine wage schedules. We are following Spence in modelling incomplete information about exogenous data, namely about types of appli- 
cants. Our modelling of incomplete information about endogenous variables, namely about applicants' actions, on the other hand, is quite different from the way the traditional literature on moral hazard has modelled unobservability, but is suited to the nature of the present setup.

In our model, the principals are the incumbents, and the agents are the job applicants. The game is played in the following sequence: (1) Each incumbent first designs a wage schedule as his strategy. (2) Each applicant then chooses an education level, and (3) finally chooses the best contract for him. Anticipating optimal reactions of the applicants in (2) and (3), the incumbents play a game in the above stage (1) (called the first-stage game) ${ }^{1}$. We analyze the first-stage game; analysis of the subsequent stages is trivial.

We consider two situations: one in which the incumbents behave noncooperatively and passively, and the other in which the incumbents behave cooperatively and passively. Associated with each situation, we propose an equilibrium concept: a noncooperative equilibrium, a version of the Nash equilibrium for the noncooperative behavioral principle, and a cooperative equilibrium, a version of the strong equilibrium for the cooperative behavioral principle. Our first observation is negative: a cooperative equilibrium does not exist. On the other hand, we obtain positive results on noncooperative equilibria; they do exist in many cases. By studying typical noncooperative equilibria, we conclude that it is not the informational advantage (defined as the abundance of measurable sets), but rather possession of the right information (in the sense that it best serves the needs of applicants) that enables an incumbent to win.

The negative result on a cooperative equilibrium is analogous to the nonexistence of a strong equilibrium in the prisoner's dilemma game. As Ichiishi and Idzik (1996) stressed, this is due to the very structure of the model (which is simplistic). For a cooperative equilibrium to exist, there have to be merits of coordination of strategies. Let $F^{S}$ be the set of all feasible strategies available to coalition $S$, coordinated and uncoordinated. There would be merits if $F^{S}$ strictly contains $\prod_{j \in S} F^{j}$. Roughly stated, however, our present model postulates that $F^{S}$ is identical to $\prod_{j \in S} F^{j}$ (apart from the informational aspect), as in the prisoner's dilemma game, hence the nonexistence result. There are countless situations in the real economy in which the above strict inclusion holds true (including the basic situations, like the pure exchange economy). We expect that future research will establish positive results on a cooperative equilibrium, given such situations.

Our conclusion that possession of the right information enables an incumbent to win appears to be robust. We expect that this can be taken as one of the general principles that prevail in most models.

${ }^{1}$ Our theory in the present paper is in line with the mechanism theory, which postulates that the uninformed move first and the informed move second, rather than the signalling game, which postulates that the informed move first and the uninformed move second. 


\section{Model}

The player set consists of the applicants, who first go through education and then look for a job, and the incumbents, who offer jobs to applicants.

There are two types of applicants, type $L$ (low quality) and type $H$ (high quality). An applicant's type is his private information. An applicant of type $t$, when employed by an incumbent, brings in to the employer the marginal revenue $r_{t}, t=L, H$. Denote by $M$ the set of possible education levels; for simplicity, we assume that $M=[0, \bar{m}]$, a nondegenerate interval. A pair $(m, w) \in M \times \mathbf{R}_{+}$then signifies the education level and the wage level of an applicant; the pair is called a contract. The preference relation of an applicant of type $t$ is defined on the contract space $M \times \mathbf{R}_{+}$, and is represented by a continuous utility function $u(\cdot \mid t): M \times \mathbf{R}_{+} \rightarrow \mathbf{R}$, which is decreasing in $m \in M$ and is increasing in $w \in \mathbf{R}_{+}$. We postulate that each applicant has a reservation wage level, $\underline{w}$, that is, he will leave this "job market" 2 if no incumbent offers a job with a wage greater than or equal to $\underline{w}$; for simplicity we assume that this level is the same regardless of a type and also regardless of an education level. Since any contract $(m, \underline{w})$ gives the worst utility level, for any contract $(m, w)$ such that $w>\underline{w}$, there exists $w^{\prime}>\underline{w}$ such that $u\left(0, w^{\prime} \mid t\right)=u(m, w \mid t)$. We postulate

$$
0 \leq \underline{w}<r_{L}<r_{H}
$$

We also postulate that the high-quality applicant can endure education more than the low-quality applicant. This is precisely formulated by the assumption that at any contract $(m, w)$ for which $w>\underline{w}$, the slope of the indifference curve of the type- $H$ applicants is lower than that of the type- $L$ applicants. In the case $u(\cdot \mid t)$ is differentiable, it means that the high-quality applicant's marginal rate of substitution of wage for education is lower than that of the low-quality applicant:

$$
\left|\frac{\frac{\partial u(m, w \mid H)}{\partial m}}{\frac{\partial u(m, w \mid H)}{\partial w}}\right|<\left|\frac{\frac{\partial u(m, w \mid L)}{\partial m}}{\frac{\partial u(m, w \mid L)}{\partial w}}\right| .
$$

There are $n_{t}$ applicants of type $t$. Set $n:=n_{L}+n_{H}$.

Let $I$ be the finite set of incumbents. An applicant's education level may serve as a signal of his type, hence set $M$ is considered a message space. However, each incumbent can observe messages only imperfectly. While he may discern a college graduate from a high school graduate, he may not be able to discern different intensities of the education that two college graduates have gone through. On the other hand, he may be able to discern different intensities, perhaps due to the personal contact he has with the faculty of a

${ }^{2}$ Although we adopt the conventional terminology of "job market" for convenience, the game played by the applicants and the incumbents is far from the neoclassical market. 
college. Thus two incumbents may have different abilities to discern education levels. Incumbent $i$ 's discerning ability is formulated as an information structure, formally defined as a finite algebra $\mathcal{M}^{i}$ on $M$; incumbent $i$ can discern education levels $m$ and $m^{\prime}$, iff there exists $A \in \mathcal{M}^{i}$ for which $m \in A$ and $m^{\prime} \notin A$. For simplicity we assume that each minimal member of $\mathcal{M}^{i}$ is of the form, $\left[m, m^{\prime}\right)$, a half-closed and half-open interval in $M$, in case $m^{\prime} \neq \bar{m}$, or of the form $\left[m^{\prime \prime}, \bar{m}\right]$.

\section{Endogenous determination of wage schedules}

We analyze the "job market" in which (1) each incumbent $i$ first decides either to stay in the "market" or to quit, and if he stays, he announces a wage schedule, $g^{i}: M \rightarrow \mathbf{R}_{+}$, which offers a job with wage level $g^{i}(m)$ to the applicants of every possible education level $m,(2)$ each applicant $j$ then chooses his education level $\tilde{m} \in M$, and (3) applicant $j$ accepts a job from among those offered to the applicants of his education level, thereby choosing his wage level from $\left\{g^{i}(\tilde{m})\right\}_{i \in I_{+}}$, where $I_{+}$is the set of all incumbents who stay in the "job market". A wage schedule is considered a mechanism.

The applicants behave noncooperatively in the above stages (2) and (3) as the Stackelberg followers. The incumbents are the Stackelberg leaders: Anticipating optimal reactions of the applicants, the incumbents play a game (with the player set $I$ ) in the above stage (1); this game will henceforth be called the first-stage game. We will analyze two situations: one in which the incumbents also behave noncooperatively, and the other in which the incumbents behave cooperatively, that is, they may merge into a larger firm and jointly design their mechanism. The overall game is, therefore, a specific instance of a multi-principal, multi-agent problem.

Our main focus here is analysis of the first-stage game. The subsequent subgame played by the applicants, (2) and (3), is trivial. Indeed, if each remaining incumbent $i\left(\in I_{+}\right)$chooses a wage schedule $g^{i}$, then the applicants of type $t$ choose education level $m_{t}$ and sign the employment contract with any of the incumbents $i_{t}$, hence receive wage $g^{i_{t}}\left(m_{t}\right)$, so that $m_{t}$ is a solution to

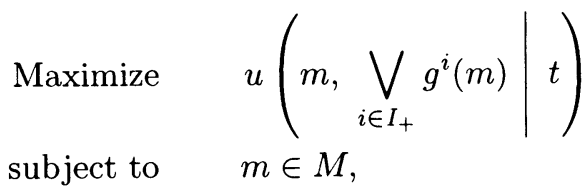

where

$$
\bigvee_{i \in I_{+}} g^{i}(m):=\max _{i \in I_{+}} g^{i}(m)
$$

and $i_{t}$ satisfies 


$$
g^{i_{t}}\left(m_{t}\right)=\bigvee_{i \in I_{+}} g^{i}\left(m_{t}\right) .
$$

A strategy of incumbent $i$ in the first-stage game is a wage-schedule $g^{i}$ : $M \rightarrow \mathbf{R}_{+}$. It is feasible if it takes the same value for any two undiscernible messages, that is, if it is $\mathcal{M}^{i}$-measurable. It keeps the applicants in the "job market" if the offered wages are no lower than the reservation wage, that is, if $g^{i}(m) \geq \underline{w}$ for all $m \in M$. An outcome of a strategy bundle $\left\{g^{i}\right\}_{i \in I_{+}}$ is the applicants' strategy-choice in the subgame (2) and (3) in accordance with the offered wage schedules $\left\{g^{i}\right\}_{i \in I_{+}}$; it is the education level $\tilde{m}_{t}$ and the number $n_{t}^{i}$ of applicants of type $t$ who accept a contract with incumbent $i$, $t \in\{L, H\}, i \in I_{+}$. The $2 \# I_{+}$nonnegative integers $\left\{n_{L}^{i}, n_{H}^{i}\right\}_{i \in I_{+}}$are called assignment. The gain of incumbent $i$ is then defined as the profit,

$$
n_{L}^{i}\left(r_{L}-g^{i}\left(\tilde{m}_{L}\right)\right)+n_{H}^{i}\left(r_{H}-g^{i}\left(\tilde{m}_{H}\right)\right) .
$$

If $i$ anticipates in the first-stage game that his gain will be negative, he will change his strategy, or else quit from the "job market". Prospect for a gain thus endogenously determines the set $I_{+}$of incumbents in the "market". The prospect in turn is determined by strategies currently chosen by the other incumbents. We postulate that when behaving noncooperatively, each incumbent is passive vis-à-vis the other incumbents' strategy-choice. Given a strategy bundle $\left\{g^{i}\right\}_{i \in I}$ with the associated assignment $\left\{n_{L}^{i}, n_{H}^{i}\right\}_{i \in I}$, incumbent $i$ is called active if $n_{L}^{i}>0$ or $n_{H}^{i}>0$. A noncooperative equilibrium of the first-stage game is an \#I-tuple of mechanisms $\left\{g^{* i}\right\}_{i \in I}$ together with the associated assignment $\left\{n_{L}^{* i}, n_{H}^{* i}\right\}_{i \in I}$ such that

- each mechanism $g^{* i}$ is feasible, keeps the applicants in the "job market", and receives a nonnegative gain; and

- it is not true that there is an incumbent who can improve upon the outcome of $\left\{g^{* i}\right\}_{i \in I}$, that is, it is not true that there are incumbent $j \in I$ and his feasible strategy $g^{j}: M \rightarrow \mathbf{R}_{+}$such that $j$ remains active and receives a nonnegative gain given strategy bundle $\left\{g^{j},\left\{g^{* i}\right\}_{i \in I \backslash\{j\}}\right\}$, and such that denoting by $I_{+}$the set of the incumbents remaining in the "market", $j$ 's gain from the remaining strategy bundle $\left\{g^{j},\left\{g^{* i}\right\}_{i \in I_{+} \backslash\{j\}}\right\}$ is greater than his gain from the outcome of the strategy bundle $\left\{g^{* i}\right\}_{i \in I}$.

As an alternative to the passive noncooperative behavior of the incumbents, we also model a passive cooperative behavior. Denote by $\mathcal{I}$ the family of nonempty coalitions of incumbents, $2^{I} \backslash\{\emptyset\}$. A coalition structure is a partition of $I$. The members of a coalition $T$ jointly design a mechanism $g^{T}: M \rightarrow \mathbf{R}_{+}$; in so doing, they can pool their private information, so $g^{T}$ is feasible if it is $\bigvee_{i \in T} \mathcal{M}^{i}$-measurable. Suppose coalition structure $\mathcal{P}$ is realized and each coalition $T \in \mathcal{P}$ chooses a wage schedule $g^{T}$. The applicants then play the subsequent subgame, in accordance with the most advantageous schedule, $\bigvee_{T \in \mathcal{P}} g^{T}$. The outcome in turn determines a gain of each coalition in $\mathcal{P}$. As a part of the first-stage game, the members of a coalition in $\mathcal{P}$ agree 
in the first stage on distribution of the anticipated coalitional gain among themselves. A cooperative equilibrium of the first-stage game is a triple of a coalition structure $\mathcal{P}^{*}$, a \# $\mathcal{P}^{*}$-tuple of mechanisms $\left\{g^{* T}\right\}_{T \in \mathcal{P}^{*}}$, and a gain distribution among the incumbents $\left\{\pi^{* i}\right\}_{i \in I}$, such that

- for each realized coalition $T \in \mathcal{P}^{*}$, its mechanism $g^{* T}$ is feasible and keeps the applicants in the "job market", and the nonnegative gain distribution is feasible, that is, $\pi^{* i} \geq 0$, and $\sum_{i \in T} \pi^{* i}$ is less than or equal to $T^{\prime}$ 's coalitional gain; and

- it is not true that there is a coalition of incumbents which can improve upon the gain distribution $\left\{\pi^{* i}\right\}_{i \in I}$, that is, it is not true that there are coalition $S \in \mathcal{I}$, its feasible strategy $g^{S}: M \rightarrow \mathbf{R}_{+}$and its feasible gain distribution $\left\{\pi^{i}\right\}_{i \in S}$, such that $\pi^{i}>\pi^{* i}$ for every $i \in S$.

Here, the second equilibrium condition (the coalitional stability condition) is ambiguous, and there are actually many precise versions. The ambiguity arises, because in analyzing the effects of a deviating coalition, we need to specify actions of the non-deviating incumbents: We postulate that when behaving cooperatively, the members of each coalition are passive vis-à-vis the other coalitions' strategy-choice. The members of a deviating coalition $S$ perceive, therefore, that those coalitions $T$ in $\mathcal{P}^{*}$ that do not lose their members to $S$ (those $T \in \mathcal{P}^{*}$ for which $T \cap S=\emptyset$ ) keep the same strategies $g^{* T}$. We need to specify, however, strategies chosen by the incumbents who lose some colleagues to $S$ (for the coalitions $T \backslash S$ for $T \in \mathcal{P}^{*}$ for which $\emptyset \neq T \backslash S \neq T$, we need to specify their strategies perceived by the members of $S$ ). There are many specifications, hence many versions of the coalitional stability condition. One scenario for the deviating coalition $S$ 's perception is that for each $T \in \mathcal{P}^{*}$, the members of $T$ who are left behind at the time of formation of $S$ stay together afterwards, that is, the coalition structure $\{S\} \bigcup\left\{T \backslash S \mid T \in \mathcal{P}^{*}, T \backslash S \neq \emptyset\right\}$ is realized as a result of formation of $S$, and that, for each $T \in \mathcal{P}^{*}$ for which $T \backslash S \neq \emptyset$, the coalition of the remaining players $T \backslash S$ keep choosing $g^{* T}$ as its feasible strategy, since each member in $T \backslash S$ has learnt the information structure $\bigvee_{i \in T} \mathcal{M}^{i}$ through the earlier cooperation of the members of $T$. The gain of each coalition that coexists after formation of $S$ is then determined by the subgame given the wage schedule:

$$
g^{S} \bigvee\left(\bigvee_{T \in \mathcal{P}^{*}: T \backslash S \neq \emptyset} g^{* T}\right) .
$$

There are other scenarios; in particular, we may allow some incumbents to leave the "job market", as we did in formulating the noncooperative equilibrium. It will turn out that the results on the cooperative equilibrium in this paper are obtained for a wide class of scenarios. The only postulate we make is: 
- Suppose that each coalition $T$ in the prevailing coalition structure $\mathcal{P}^{*}$ is choosing strategy $g^{* T}$, and that coalition $S$ is formed against $\mathcal{P}^{*}$ and chooses strategy $g^{S}$. Then, coalition $S$ can attract all the applicants who have education level $m$, only if $g^{S}(m)>\bigvee_{T \in \mathcal{P}^{*}: T \backslash S \neq \emptyset} g^{* T}(m)$.

In the following analysis, we will concentrate on the nontrivial case of multi-principals, $\# I \geq 2$. In the trivial case of $\# I=1$, say $I=\{i\}$, the wage schedule $g^{* i}: m \mapsto \underline{w}$ is an equilibrium.

We first state a basic negative result on the cooperative equilibrium:

Proposition 3.1. Assume \#I $\geq 2$. If the grand coalition $I$ and the singleton coalitions can form, then there is no cooperative equilibrium.

The rest of this section is devoted to study of the noncooperative equilibrium. We establish existence results for several cases by constructing specific noncooperative equilibria. The specific formula of equilibria provides an insight into the role that information structures $\mathcal{M}^{i}, i \in I$, play in the "job market".

Define $w_{0} \in \mathbf{R}_{+}$by

$$
n_{L}\left(r_{L}-w_{0}\right)+n_{H}\left(r_{H}-w_{0}\right)=0,
$$

and let $U_{H 0}$ be the indifference curve of the type- $H$ applicants that passes through the contract $\left(0, w_{0}\right)$. See figure 1 .

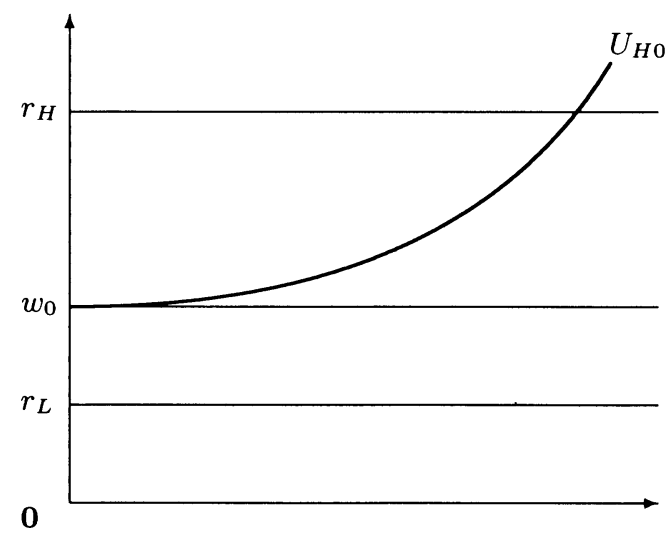

Figure 1. definitions of $w_{0}$ and $U_{H 0}$

Let $U_{t}$ be an indifference curve of the type- $t$ applicants, $t=H, L$. By abuse of notation, $U_{t}$ and $r_{t}$ also denote the functions from $M$ to $\mathbf{R}_{+}$whose graphs are $U_{t}$ and the horizontal line of height $r_{t}$, respectively. Thus, $(m, w) \in$ $U_{t}$ iff $w=U_{t}(m)$. The function $U_{L} \wedge r_{H}: M \rightarrow \mathbf{R}_{+}$is then defined by $\left(U_{L} \bigwedge r_{H}\right)(m):=\min \left\{U_{L}(m), r_{H}(m)\right\}$. 
For $h=1,2, \ldots$, define $m_{h} \in M$ so that $\left[0, m_{h}\right)$ is the $h$ th smallest nondegenerate interval that can be distinguished by some incumbents. Clearly,

$$
0<m_{1}<m_{2}<\ldots .
$$

For each $h$, choose $w_{L, h}$ and $w_{H, h}$ so that

$$
\begin{aligned}
n_{L}\left(r_{L}-w_{L, h}\right)+n_{H}\left(r_{H}-w_{H, h}\right) & =0 \\
u\left(0, w_{L, h} \mid L\right) & =u\left(m_{h}, w_{H, h} \mid L\right) .
\end{aligned}
$$

The pair $\left(w_{L, h}, w_{H, h}\right)$ is uniquely determined. Denote by $U_{L, h}$ the indifference curve of the type- $L$ applicants that passes through $\left(0, w_{L, h}\right)$ and $\left(m_{h}, w_{H, h}\right)$. Denote also by $U_{H, h}$ the indifference curve of the type- $H$ applicants that passes through $\left(m_{h}, w_{H, h}\right)$. See figure 2. Let $I_{h}$ be the set of all incumbents who can distinguish the interval $\left[0, m_{h}\right) ; I_{h}:=\left\{i \in I \mid\left[0, m_{h}\right) \in \mathcal{M}^{i}\right\}$.

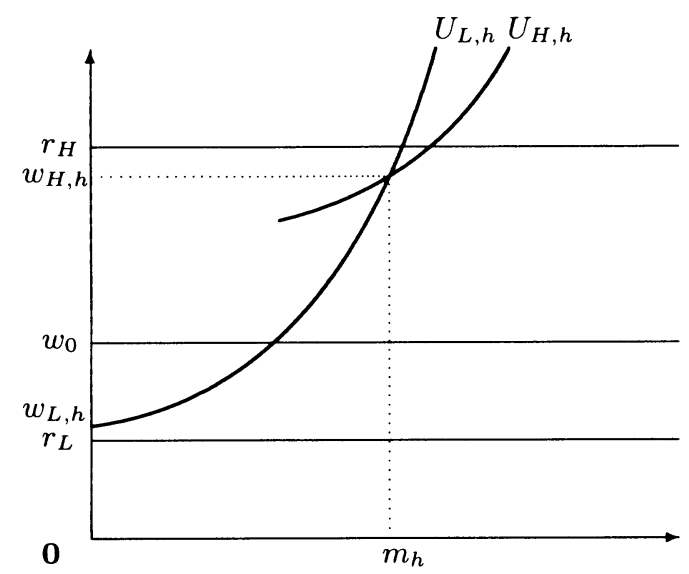

Figure 2. definitions of $w_{H, h}, w_{L, h}, U_{H, h}$ and $U_{L, h}$

Let $k$ be the positive integer for which $u\left(m_{k}, w_{H, k} \mid H\right)$ is the highest, that is,

$$
u\left(m_{k}, w_{H, k} \mid H\right) \geq u\left(m_{h}, w_{H, h} \mid H\right), \text { for all } h .
$$

If there is a tie, choose $k$ so that $w_{L, k}$ is the highest among such maximizers of $u\left(m_{h}, w_{H, h} \mid H\right)$ (or equivalently, $m_{k}$ is the smallest among such maximizers).

Any incumbent in $I_{h}$ can technically offer two contracts, $\left(0, w_{L, h}\right)$ and $\left(m_{h}, w_{H, h}\right)$. When incumbent $i \in I_{h}$ offers these two contracts, and no other contracts are available in the "job market", the type- $H$ applicants sign contract $\left(m_{h}, w_{H, h}\right)$, the type- $L$ applicants are willing to sign contract $\left(0, w_{L, h}\right)$, and $i$ receives zero profit. The type- $H$ applicants find $\left(m_{k}, w_{H, k}\right)$ the most desirable of the contracts

$$
\bigcup\left\{\left(0, w_{L, h}\right),\left(m_{h}, w_{H, h}\right) \mid h=1,2, \ldots\right\},
$$


so the incumbents $I_{k}$ are in an advantageous position in attracting them. It will appear that the contracts $\left(0, w_{L, k}\right)$ and $\left(m_{k}, w_{H, k}\right)$ are actually established as equilibrium contracts in several important situations (like subcases (2.1) and (2.2) below), so the incumbents $I_{k}$ possess the right information to win the noncooperative game in these situations.

For the required existence results, we need to consider several mutually exclusive and exhaustive cases:

$$
\begin{aligned}
& \text { Case (1): } \quad w_{H, k} \leq U_{H 0}\left(m_{k}\right), \\
& \text { Case (2): } \quad r_{H} \geq w_{H, k}>U_{H 0}\left(m_{k}\right), \\
& \text { Case (3): } \quad r_{H}<w_{H, k}, \text { and } w_{H, k}>U_{H 0}\left(m_{k}\right) .
\end{aligned}
$$

Notice that $w_{L, k} \geq r_{L}\left(w_{L, k}<r_{L}\right.$, resp.) in case (2) (in case (3), resp.).

Case (1) says that the type- $H$ applicants find contract $\left(0, w_{0}\right)$ more attractive than any contract $\left(m_{h}, w_{h}\right), h=1,2, \ldots$; in other words, using the strategy

$$
g(m):= \begin{cases}w_{L, h} & \text { if } m<m_{h} \\ w_{H, h} & \text { if } m \geq m_{h}\end{cases}
$$

nobody can improve upon the constant strategy $m \mapsto w_{0}$. The following Theorem 3.2 establishes that in case (1) we have the result stronger than the mere definition (of case (1)): that using whatever feasible strategy no incumbent can improve upon the constant strategy $m \mapsto w_{0}$.

Theorem 3.2. Assume \#I $\geq 2$, and consider case (1). Strategy bundle $\left\{g^{* i}\right\}_{i \in I}$ is a noncooperative equilibrium, if

$$
g^{* i}(m)=w_{0}, \text { for all } i \in I \text { and all } m \in M .
$$

Any assignment $\left\{n_{L}^{i}, n_{H}^{i}\right\}_{i \in I}$ may prevail with this equilibrium, provided that the gain of each incumbent is zero,

$$
n_{L}^{i}\left(r_{L}-w_{0}\right)+n_{H}^{i}\left(r_{H}-w_{0}\right)=0, \text { for all } i \in I .
$$

Case (2) is divided into three subcases:

Subcase (2.1): $\# I_{k} \geq 2$,

Subcase (2.2): $\# I_{k}=1$, say $I_{k}=\left\{i_{k}\right\}$, there is a tie in obtaining $\max _{h} u\left(m_{h}, w_{H, h} \mid H\right)$, that is, there is $k^{\prime}(\neq k)$ such that $u\left(m_{k^{\prime}}, w_{H, k^{\prime}} \mid H\right)=u\left(m_{k}, w_{H, k} \mid H\right)$, and for at least one such $k^{\prime}, I_{k^{\prime}} \backslash\left\{i_{k}\right\} \neq \emptyset$

Subcase (2.3): \# $I_{k}=1$, say $I_{k}=\left\{i_{k}\right\}$, and for any $i \in I \backslash\left\{i_{k}\right\}$ and any $h$ for which $I_{h} \ni i, u\left(m_{h}, w_{H, h} \mid H\right)<u\left(m_{k}, w_{H, k} \mid H\right)$. 
See figure 3 .

Cases (2) and (3) say that the type- $H$ applicants find contract $\left(m_{k}, w_{H, k}\right)$ more attractive than contract $\left(0, w_{0}\right)$. Subcases $(2.1)$ and $(2.2)$ say that more than one incumbent can offer the pair, $\left(0, w_{L, k}\right)$ and $\left(m_{k}, w_{H, k}\right)$, or a pair, $\left(0, w_{L, k^{\prime}}\right)$ and $\left(m_{k^{\prime}}, w_{H, k^{\prime}}\right)$, for which $\left(m_{k^{\prime}}, w_{H, k^{\prime}}\right)$ is as attractive as $\left(m_{k}, w_{H, k}\right)$. In these subcases, the members of $I_{k} \cup I_{k^{\prime}}$ are competing with each other for type- $H$ applicants, so while they can offer contracts actually signed in equilibrium, their profit is zero; this is the essential implication of the following Theorems 3.3 and 3.4. On the other hand, subcase (2.3) says that the sole member $i_{k}$ of $I_{k}$ is in the advantageous position that none of the other contracts $\left(m_{h}, w_{H, h}\right), h \neq k$, are as attractive as $\left(m_{k}, w_{H, k}\right)$. Theorem 3.5 implies that $i_{k}$ enjoys this monopoly power and earns a positive profit in equilibrium.

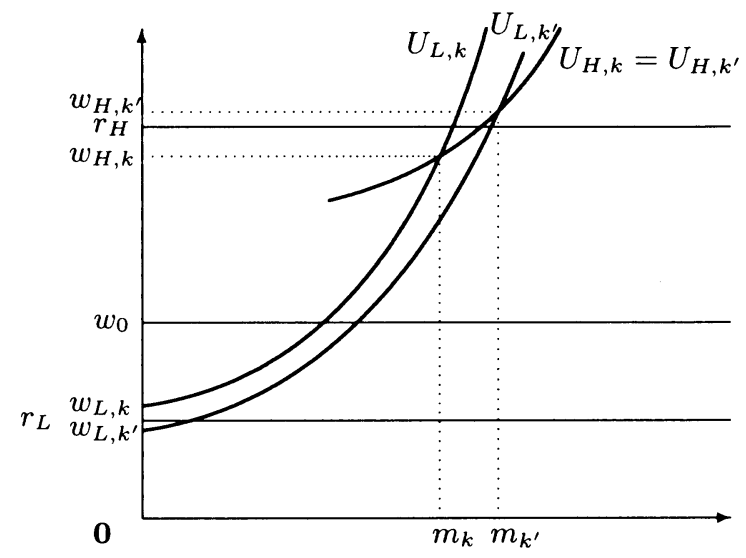

Figure 3. subcase (2.2)

Theorem 3.3. Consider subcase (2.1). Then, feasible strategy bundle $\left\{g^{* i}\right\}_{i \in I}$ is a noncooperative equilibrium, if

$$
g^{* i}(\cdot) \leq\left(U_{L, k} \bigwedge U_{H, k}\right)(\cdot), \text { for all } i \in I,
$$

and for at least two distinct members $i_{1}$ and $i_{2}$ in $I_{k}$,

$$
g^{* i_{1}}(m)=g^{* i_{2}}(m)=\left\{\begin{array}{lll}
w_{L, k} & \text { if } & m=0 \\
w_{H, k} & \text { if } & m=m_{k} .
\end{array}\right.
$$

Given this $\left\{g^{* i}\right\}_{i \in I}$, the applicants of type $L$ sign the contract $\left(0, w_{L, k}\right)$, and the applicants of type $H$ sign the contract $\left(m_{k}, w_{H, k}\right)$. Any assignment $\left(n_{L}^{i}, n_{H}^{i}\right)$ may prevail to those incumbents $i \in I_{k}$ for whom $g^{* i}(0)=w_{L, k}$ and $g^{* i}\left(m_{k}\right)=w_{H, k}$, provided that $i$ 's gain is zero, i.e., $n_{L}^{i} / n_{H}^{i}=n_{L} / n_{H}$. 
Theorem 3.4. Consider subcase (2.2). Feasible strategy bundle $\left\{g^{* i}\right\}_{i \in I}$ is a noncooperative equilibrium, if

$$
\begin{gathered}
g^{* i}(\cdot) \leq\left(U_{L, k} \bigwedge U_{H, k}\right)(\cdot), \text { for all } i \in I, \\
g^{* i_{k}}(m)=\left\{\begin{array}{lll}
w_{L, k} & \text { if } & m=0 \\
w_{H, k} & \text { if } & m=m_{k},
\end{array}\right.
\end{gathered}
$$

and for some $k^{\prime}(\neq k)$ for which $u\left(m_{k^{\prime}}, w_{H, k^{\prime}} \mid H\right)=u\left(m_{k}, w_{H, k} \mid H\right)$,

$$
\exists i^{\prime} \in I_{k^{\prime}} \backslash\left\{i_{k}\right\}: g^{* i^{\prime}}(m)=\left\{\begin{array}{lll}
w_{L, k} & \text { if } & m=0 \\
w_{H, k^{\prime}} & \text { if } & m=m_{k^{\prime}}
\end{array}\right.
$$

Given this $\left\{g^{* i}\right\}_{i \in I}$, the applicants of type $L$ sign $i_{k}$ 's contract $\left(0, w_{L, k}\right)$, and the applicants of type $H$ sign $i_{k}$ 's contract $\left(m_{k}, w_{H, k}\right)$.

For subcase (2.3), let $l$ be any positive integer such that $u\left(m_{l}, w_{H, l} \mid H\right)$ is the highest level achievable by the incumbents other than $i_{k}$, i.e.,

$$
u\left(m_{l}, w_{H, l} \mid H\right)=\max \left\{u\left(m_{h}, w_{H, h} \mid H\right) \mid I_{h} \backslash\left\{i_{k}\right\} \neq \emptyset\right\},
$$

and denote by $U_{H, l}$ the indifference curve of the type- $H$ applicants that passes through $\left(m_{l}, w_{H, l}\right)$. For each $h$, let $U_{L, h}^{\prime}$ be the indifference curve of the type- $L$ applicants that passes through $\left(m_{h}, U_{H, l}\left(m_{h}\right)\right)$, and set $w_{L, h}^{\prime}:=U_{L, h}^{\prime}(0)$. Let $U_{L, h}^{\prime \prime}$ be the indifference curve of the type- $L$ applicants that passes through $\left(0, \max \left\{r_{L}, w_{L, h}^{\prime}\right\}\right)$. See figure 4 . Let $J_{i_{k}}$ be the set of all integers $h$ such that the interval $\left[0, m_{h}\right)$ is discernable to incumbent $i_{k},\left\{h \mid I_{h} \ni i_{k}\right\}$. We are going to compare mechanisms parametrized by $h \in J_{i_{k}}$, so that the mechanism for $h$ is intended to offer contract $\left(0, \max \left\{r_{L}, w_{L, h}^{\prime}\right\}\right)$ to type- $L$ applicants and contract $\left(m_{h}, U_{H, l}\left(m_{h}\right)\right)$ to type- $H$ applicants. Let $k^{*} \in J_{i_{k}}$ be the parameter that maximizes $i_{k}$ 's gain, of all such parametrized mechanisms: the parameter $k^{*}$ solves

$$
\begin{array}{ll}
\text { Maximize } & n_{L}\left(r_{L}-\max \left\{r_{L}, w_{L, h}^{\prime}\right\}\right)+n_{H}\left(r_{H}-U_{H, l}\left(m_{h}\right)\right), \\
\text { subject to } & h \in J_{i_{k}} .
\end{array}
$$

Set $w_{L}^{\prime *}:=w_{L, k^{*}}^{\prime}, U_{L}^{\prime *}:=U_{L, k^{*}}^{\prime}$, and $U_{L}^{\prime *}:=U_{L, k^{*}}^{\prime \prime}$

Theorem 3.5. Assume \#I $\geq 3$, and consider subcase (2.3). Assume that there exists an integer $p$ for which $m_{k^{*}} \leq m_{p}$, and $I_{p} \backslash\left\{i_{k}\right\} \neq \emptyset$. Feasible strategy bundle $\left\{g^{* i}\right\}_{i \in I}$ is a noncooperative equilibrium, if

$$
\begin{gathered}
g^{* i}(\cdot) \leq\left(U_{L}^{\prime \prime *} \bigwedge U_{H, l}\right)(\cdot), \text { for all } i \in I, \\
g^{* i_{k}}(m)=\left\{\begin{array}{lll}
\max \left\{r_{L}, w_{L}^{\prime *}\right\} & \text { if } m=0 \\
U_{H, l}\left(m_{k^{*}}\right) & \text { if } m=m_{k^{*}},
\end{array}\right. \\
\exists i_{p} \in I_{p} \backslash\left\{i_{k}\right\}: g^{* i_{p}}(m)=\left\{\begin{array}{lll}
\max \left\{r_{L}, w_{L}^{\prime *}\right\} & \text { if } m=0 \\
U_{H, l}\left(m_{p}\right) & \text { if } m=m_{p},
\end{array}\right.
\end{gathered}
$$




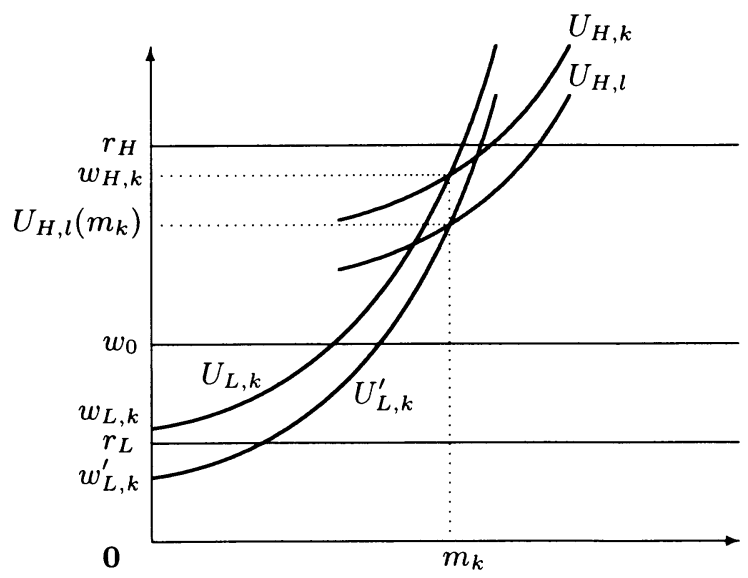

Figure 4. subcase (2.3)

and

$$
\exists i^{\prime} \in I \backslash\left\{i_{k}, i_{p}\right\}: g^{* i^{\prime}}(m)=r_{L} \text { for all } m \in M .
$$

Given this $\left\{g^{* i}\right\}_{i \in I}$, the applicants of type $L$ sign $i_{k}$ 's contract $\left(0, \max \left\{r_{L}, w_{L}^{\prime *}\right\}\right)$, and the applicants of type $H$ sign $i_{k}$ 's contract $\left(m_{k^{*}}, H_{H, l}\left(m_{k^{*}}\right)\right)$.

Remark 3.6. A typical equilibrium for subcase (2.1) is given by: for all $i \in I_{k}$,

$$
g^{* i}(m)=\left\{\begin{array}{lll}
w_{L, k} & \text { if } & 0 \leq m<m_{k} \\
w_{H, k} & \text { if } \quad m_{k} \leq m
\end{array}\right.
$$

In subcase (2.2), incumbent $i_{k}$ seemingly has a strict informational advantage since $w_{L, k}>w_{L, k^{\prime}}$, but by adopting the strategy $g^{i_{k}}$ defined by

$$
g^{i_{k}}(m)=\left\{\begin{array}{lll}
w_{L, k^{\prime}} & \text { if } & 0 \leq m<m_{k} \\
w_{H, k} & \text { if } \quad m_{k} \leq m
\end{array}\right.
$$

he could not separate applicants of different types (all applicants would sign the contract $\left.\left(m_{k}, w_{H, k}\right)\right)$. A typical equilibrium for subcase (2.3) is given by

$$
g^{* i_{k}}(m)=\left\{\begin{array}{lll}
\max \left\{r_{L}, w_{L, k}^{\prime}\right\} & \text { if } & 0 \leq m<m_{k} \\
U_{H, l}\left(m_{k}\right) & \text { if } \quad m_{k} \leq m .
\end{array}\right.
$$

The equilibrium of Theorem 3.2 is a pooling equilibrium. The equilibria of Theorems 3.3-3.5 are separating equilibria.

In order to analyze case (3), define for each $h=1,2, \ldots$,

$$
\begin{aligned}
& \underline{w}_{H, h}:=\min \left\{r_{H}, w_{H, h}\right\}, \\
& \bar{w}_{L, h}:=\max \left\{r_{L}, w_{L, h}\right\},
\end{aligned}
$$

and denote by $\underline{U}_{H, h}\left(\bar{U}_{L, h}\right.$, resp.) the indifference curve of the type- $H$ applicants (of the type- $L$ applicants, resp.) that passes through $\left(m_{h}, \underline{w}_{H, h}\right)$ 
(through $\left(0, \bar{w}_{L, h}\right)$, resp.) Notice that $\underline{w}_{H, h}=w_{H, h}$ iff $\bar{w}_{L, h}=w_{L, h}$, and that $u\left(0, \bar{w}_{L, h} \mid L\right) \geq u\left(m_{h}, \underline{w}_{H, h} \mid L\right)$. Re-define $k$ as the positive integer for which $u\left(m_{k}, \underline{w}_{H, k} \mid H\right)$ is the highest, that is,

$$
u\left(m_{k}, \underline{w}_{H, k} \mid H\right) \geq u\left(m_{h}, \underline{w}_{H, h} \mid H\right), \text { for all } h .
$$

If there is a tie, choose $k$ so that $\bar{w}_{L, k}$ is the highest among such maximizers of $u\left(m_{h}, \underline{w}_{H, h} \mid H\right)$. We consider mutually exclusive and exhaustive subcases:

Subcase (3.1): $\underline{w}_{H, k} \leq U_{H 0}\left(m_{k}\right)$,

Subcase (3.2): $\underline{w}_{H, k}>U_{H 0}\left(m_{k}\right)$.

By definition, $r_{H} \geq \underline{w}_{H, k}$. Subcase (3.2) is divided into three subsubcases:

Subcase (3.2.1): $\# I_{k} \geq 2$,

Subcase (3.2.2): \# $I_{k}=1$, say $I_{k}=\left\{i_{k}\right\}$, and there is $k^{\prime}(\neq k)$ such that $u\left(m_{k^{\prime}}, \underline{w}_{H, k^{\prime}} \mid H\right)=u\left(m_{k}, \underline{w}_{H, k} \mid H\right)$ and $I_{k^{\prime}} \backslash\left\{i_{k}\right\} \neq \emptyset$.

Subcase (3.2.3): \# $I_{k}=1$, say $I_{k}=\left\{i_{k}\right\}$, and for any $i \in I \backslash\left\{i_{k}\right\}$ and any $h$ for which $I_{h} \ni i, u\left(m_{h}, \underline{w}_{H, h} \mid H\right)<u\left(m_{k}, \underline{w}_{H, k} \mid H\right)$.

For subcase (3.2.3), define $\underline{U}_{H, l}, k^{*}, \underline{w}_{L}^{\prime *}, \underline{U}_{L}^{\prime *}$ and $\underline{U}_{L}^{\prime *}$ as in subcase $(2.3)^{3}$. The next theorem says that in case (3), essentially the same conclusions as in cases (1)-(2) hold true by substituting $\underline{w}_{H, k}, \bar{w}_{L, k}, \underline{U}_{H, k}, \bar{U}_{L, k}, \underline{U}_{H, l}, \underline{w}_{L}^{\prime *}$, $\underline{U}_{L}^{\prime *}$ and $\underline{U}_{L}^{\prime \prime *}$ for $w_{H, k}, w_{L, k}, U_{H, k}, U_{L, k}, U_{H, l}, w_{L}^{\prime *}, U_{L}^{\prime *}$ and $U_{L}^{\prime *}$, respectively.

Theorem 3.7. Consider case (3), and let $\left\{g^{* i}\right\}_{i \in I}$ be a feasible strategy bundle.

(i) In subcase (3.1), suppose \#I $\geq 2$, and

$$
g^{* i}(m)=w_{0}, \quad \text { for all } i \in I \text { and all } m \in M \text {. }
$$

(ii) In subcases (3.2.1) and (3.2.2), suppose

$$
g^{* i}(\cdot) \leq\left(\bar{U}_{L, k} \bigwedge \underline{U}_{H, k}\right)(\cdot), \text { for all } i \in I .
$$

(iii) In subcase (3.2.1), suppose for at least two distinct members $i_{1}$ and $i_{2}$ in $I_{k}$,

${ }^{3}$ The number $l$ is defined as any positive integer such that $u\left(m_{l}, \underline{w}_{H, l} \mid H\right)$ is the highest level achievable by the incumbents other than $i_{k}, \underline{U}_{H, l}$ is the indifference curve of the type- $H$ applicants that passes through $\left(m_{l}, \underline{w}_{H, l}\right)$. For each $h$, $\underline{U}_{L, h}^{\prime}$ is the indifference curve of the type- $L$ applicants that passes through $\left(m_{h}, \underline{U}_{H, l}\left(m_{h}\right)\right), \underline{w}_{L, h}^{\prime}$ is defined as $\underline{U}_{L, h}^{\prime}(0)$, and $\underline{U}_{L, h}^{\prime \prime}$ is the indifference curve of the type- $L$ applicants that passes through $\left(0, \max \left\{r_{L}, \underline{w}_{L, h}^{\prime}\right\}\right)$. The integer $k^{*}$ is the specific $h \in J_{i_{k}}$ at which $n_{L}\left(r_{L}-\max \left\{r_{L}, \underline{w}_{L, h}^{\prime}\right\}\right)+n_{H}\left(r_{H}-\underline{U}_{H, l}\left(m_{h}\right)\right)$ is maximized. Then, $\underline{w}_{L}^{\prime *}:=\underline{w}_{L, k^{*}}^{\prime}, \underline{U}_{L}^{\prime *}:=\underline{U}_{L, k^{*}}^{\prime}$, and $\underline{U}_{L}^{\prime *}:=\underline{U}_{L, k^{*}}^{\prime \prime}$. 


$$
g^{* i_{1}}(m)=g^{* i_{2}}(m)=\left\{\begin{array}{lll}
\bar{w}_{L, k} & \text { if } & m=0 \\
\underline{w}_{H, k} & \text { if } & m=m_{k} .
\end{array}\right.
$$

(iv) In subcase (3.2.2), suppose

$$
g^{* i_{k}}(m)=\left\{\begin{array}{lll}
\bar{w}_{L, k} & \text { if } & m=0 \\
\underline{w}_{H, k} & \text { if } & m=m_{k},
\end{array}\right.
$$

and for some $k^{\prime}(\neq k)$ for which $u\left(m_{k^{\prime}}, \underline{w}_{H, k^{\prime}} \mid H\right)=u\left(m_{k}, \underline{w}_{H, k} \mid H\right)$,

$$
\begin{gathered}
\exists i_{k^{\prime}} \in I_{k^{\prime}} \backslash\left\{i_{k}\right\}: g^{* i_{k^{\prime}}}(m)=\left\{\begin{array}{lll}
\bar{w}_{L, k} & \text { if } & m=0 \\
\underline{w}_{H, k^{\prime}} & \text { if } & m=m_{k^{\prime}}
\end{array}\right. \\
\exists i^{\prime} \in I \backslash\left\{i_{k}, i_{k^{\prime}}\right\}: g^{* i^{\prime}}(m)=r_{L} \text { for all } m \in M .
\end{gathered}
$$

(v) In subcase (3.2.3), suppose that there exists an integer $p$ for which $m_{k^{*}} \leq$ $m_{p}$, and $I_{p} \backslash\left\{i_{k}\right\} \neq \emptyset$, and that

$$
\begin{gathered}
g^{* i}(\cdot) \leq\left(\underline{U}_{L}^{\prime *} \bigwedge \underline{U}_{H, l}\right)(\cdot), \text { for all } i \in I, \\
g^{* i_{k}}(m)=\left\{\begin{array}{lll}
\max \left\{r_{L}, \underline{w}_{L}^{\prime *}\right\} & \text { if } m=0 \\
\underline{U}_{H, l}\left(m_{k^{*}}\right) & \text { if } m=m_{k^{*}},
\end{array}\right. \\
\exists i_{p} \in I_{p} \backslash\left\{i_{k}\right\}: g^{* i_{p}}(m)=\left\{\begin{array}{lll}
\max \left\{r_{L}, \underline{w}_{L}^{\prime *}\right\} & \text { if } m=0 \\
\underline{U}_{H, l}\left(m_{p}\right) & \text { if } m=m_{p},
\end{array}\right. \\
\exists i^{\prime} \in I \backslash\left\{i_{k}, i_{p}\right\}: g^{* i^{\prime}}(m)=r_{L} \text { for all } m \in M .
\end{gathered}
$$

Then, $\left\{g^{* i}\right\}_{i \in I}$ is a noncooperative equilibrium.

The final proposition in this paper is intended to be the first step towards characterizing the noncooperative equilibria. Let $k$ be the positive integer for which $u\left(m_{k}, w_{H, k} \mid H\right)$ is the highest (this definition is the same as before for cases (1) and (2), but is different from the earlier definition for case (3)).

Proposition 3.8. Let $\left\{g^{i}\right\}_{i \in I}$ be a noncooperative equilibrium. Then,

$$
g^{i}(\cdot) \leq\left(U_{H 0} \bigvee U_{H, k}\right)(\cdot), \text { for all } i \in I
$$

\section{Proofs}

Proof of Proposition 3.1. Choose any coalition structure $\overline{\mathcal{P}}$ and any feasible strategy $\bar{g}^{T}$ for each $T \in \overline{\mathcal{P}}$ which keeps the applicants on the "job market", and define $\bar{g}:=\bigvee_{T \in \overline{\mathcal{P}}} \bar{g}^{T}$. Let $\bar{m}_{t}$ be the message that the applicants of type $t$ send, given $\bar{g}$, and let $\bar{\pi}_{i}$ be a gain distributed to incumbent $i$. We need to show that some coalition improves upon $\left(\overline{\mathcal{P}},\left\{\bar{g}^{T}\right\}_{T \in \mathcal{P}},\left\{\bar{\pi}_{i}\right\}_{i \in I}\right)$. 
If $\bar{g}\left(\bar{m}_{t}\right)>\underline{w}$ for some type $t \in\{L, H\}$, then

$$
\begin{aligned}
\sum_{i \in I} \bar{\pi}_{i} & \leq n_{L}\left(r_{L}-\bar{g}\left(\bar{m}_{L}\right)\right)+n_{H}\left(r_{H}-\bar{g}\left(\bar{m}_{H}\right)\right) \\
& <n_{L}\left(r_{L}-\underline{w}\right)+n_{H}\left(r_{H}-\underline{w}\right) .
\end{aligned}
$$

So the grand coalition $I$ can improve by adopting $g^{I}: m \mapsto \underline{w}$.

If on the other hand $\bar{g}\left(\bar{m}_{t}\right)=\underline{w}$ for each type $t \in\{L, H\}$,

$$
\sum_{i \in I} \bar{\pi}_{i} \leq n_{L}\left(r_{L}-\underline{w}\right)+n_{H}\left(r_{H}-\underline{w}\right) \text {. }
$$

If strict inequality holds true here, then the grand coalition can improve by adopting $\bar{g}$ and a more efficient gain distribution. So assume that equality holds true. Then, there exists $i_{0} \in I$ for whom $\bar{\pi}_{i_{0}}>0$, and consequently for each $i \in I \backslash\left\{i_{0}\right\}(\neq \emptyset)$,

$$
\bar{\pi}_{i} \leq n_{L}\left(r_{L}-\underline{w}\right)+n_{H}\left(r_{H}-\underline{w}\right)-\bar{\pi}_{i_{0}} .
$$

Therefore the singleton $\{i\}$ forms, adopts strategy $g^{i}: m \mapsto \underline{w}+\varepsilon$, where $\varepsilon$ is a positive real number, attracts all the applicants, and obtains the entire gain

$$
n_{L}\left(r_{L}-\underline{w}-\varepsilon\right)+n_{H}\left(r_{H}-\underline{w}-\varepsilon\right) .
$$

For $\varepsilon$ small enough, this gain is greater than $\bar{\pi}_{i}$.

In order to prove Theorems 3.2-3.5 and 3.7, we first establish lemmas:

Lemma 4.1. Assume \#I $\geq 2$. Let $\left\{g^{* i}\right\}_{i \in I}$ be a noncooperative equilibrium, and let $\left\{n_{L}^{* i}, n_{H}^{* i}\right\}_{i \in I}$ be the associated assignment. For $i \in I$ and $t \in\{L, H\}$ for which $n_{t}^{* i}>0$, let $\left(m_{t}^{* i}, g^{* i}\left(m_{t}^{* i}\right)\right)$ be $i$ 's offered contract that is signed by type-t applicants.

(i) If $n_{L}^{* i}>0$, then $g^{* i}\left(m_{L}^{* i}\right) \geq r_{L}$.

(ii) If $n_{L}^{* i}>0$, then $m_{L}^{* i}=0$.

(iii) If $n_{L}^{* i}>0$ and $n_{L}^{* j}>0$, then $g^{* i}(0)=g^{* j}(0)$.

(iv) If $n_{H}^{* i}>0$, then $g^{* i}\left(m_{H}^{* i}\right) \leq r_{H}$.

Proof. (i) Suppose the contrary, i.e., suppose

$$
\exists i_{0}: n_{L}^{* i_{0}}>0, \text { and } g^{* i_{0}}\left(m_{L}^{* i_{0}}\right)<r_{L}
$$

Denote by $U_{L}^{*}$ the indifference curve of the type- $L$ applicants that passes through the contract $\left(m_{L}^{* i_{0}}, g^{* i_{0}}\left(m_{L}^{* i_{0}}\right)\right)$. Notice that $g^{* i_{0}}\left(m_{L}^{* i_{0}}\right) \geq U_{L}^{*}(0)$, and that the equality holds true iff $m_{L}^{* i_{0}}=0$. Choose any $\varepsilon>0$, and define $w_{\varepsilon}:=U_{L}^{*}(0)+\varepsilon$. Choose any $i \in I \backslash\left\{i_{0}\right\}$, and consider $i$ 's strategy $g_{\varepsilon}^{i}$ defined by

$$
g_{\varepsilon}^{i}(m):=\max \left\{g^{* i}(m), w_{\varepsilon}\right\}
$$


It suffices to show that $i$ can take away applicants from $i_{0}$ and improve upon the outcome of $\left\{g^{* j}\right\}_{j \in I}$. Since $g^{* i}$ is $\mathcal{M}^{i}$-measurable, so is $g_{\varepsilon}^{i}$. Since $U_{L}^{*}(m) \geq g^{* i}(m)$ for all $m$, it follows that

$$
g_{\varepsilon}^{i}(0)=w_{\varepsilon} .
$$

If $i$ changes his strategy from $g^{* i}$ to $g_{\varepsilon}^{i}$ while the other incumbents $h$ keep their strategies $g^{* h}$, all type- $L$ applicants will choose $i$ 's new contract $\left(0, w_{\varepsilon}\right)$, since

$$
\forall m: u\left(0, w_{\varepsilon} \mid L\right)>u\left(m_{L}^{* i_{0}}, g^{* i_{0}}\left(m_{L}^{* i_{0}}\right) \mid L\right) \geq u\left(m, g^{* h}(m) \mid L\right) .
$$

So $i$ increases his gain from the type- $L$ applicants at least by

$$
\begin{cases}\left(n_{L}-n_{L}^{* i}\right)\left(r_{L}-w_{\varepsilon}\right)+n_{L}^{* i}(-\varepsilon), & \text { if } \quad n_{L}^{* i}>0 \\ n_{L}\left(r_{L}-w_{\varepsilon}\right), & \text { if } \quad n_{L}^{* i}=0\end{cases}
$$

If, on the one hand, all type- $H$ applicants also choose $i$ 's contract $\left(0, w_{\varepsilon}\right)$, $i$ increases his gain from the type- $H$ applicants at least by $n_{H}(-\varepsilon)$, since $g^{j}\left(m_{H}^{* j}\right) \geq U_{L}^{*}(0)$ for all $j$ for which $n_{H}^{j}>0$. If, on the other hand, no applicant of type $H$ switches his contract, then $i$ 's gain from the type- $H$ applicants remains constant. Therefore, by changing a strategy from $g^{* i}$ to $g_{\varepsilon}^{i}, i$ increases his gain at least by

$$
\left\{\begin{array}{lll}
\left(n_{L}-n_{L}^{* i}\right)\left(r_{L}-w_{\varepsilon}\right)+\left(n_{L}^{* i}+n_{H}\right)(-\varepsilon), & \text { if } \quad n_{L}^{* i}>0 \\
n_{L}\left(r_{L}-w_{\varepsilon}\right)+n_{H}(-\varepsilon), & \text { if } \quad n_{L}^{* i}=0
\end{array}\right.
$$

Since $n_{L}-n_{L}^{* i} \geq n_{L}^{* i_{0}}>0, i$ strictly increases his gain for all $\varepsilon$ sufficiently close to 0 , contradicting the definition of $\left\{g^{* h}\right\}_{h \in I}$ as a noncooperative equilibrium.

(ii) Suppose the contrary, i.e., suppose

$$
\exists i_{0}: n_{L}^{* i_{0}}>0, \text { and } m_{L}^{* i_{0}}>0 .
$$

Let $U_{L}^{*}$ be the indifference curve of the type- $L$ applicants that passes through the contract $\left(m_{L}^{* i_{0}}, g^{* i_{0}}\left(m_{L}^{* i_{0}}\right)\right)$. For each type $t \in\{L, H\}$, let $I_{t}^{*}$ be the set of incumbents whose contract is signed by type- $t$ applicants, $\left\{i \in I \mid n_{t}^{* i}>0\right\}$.

Since all type- $L$ applicants sign contracts on $U_{L}^{*}$,

$$
\begin{aligned}
g^{* i}\left(m_{L}^{* i}\right) & \geq U_{L}^{*}(0) \text { for all } i \in I_{L}^{*}, \\
g^{* i_{0}}\left(m_{L}^{* i_{0}}\right) & >U_{L}^{*}(0) .
\end{aligned}
$$

Let $k \in I_{H}^{*}$ be the incumbent whose contract signed by type- $H$ applicants requires the least amount of education:

$$
\forall i \in I_{H}^{*}: m_{H}^{* k} \leq m_{H}^{* i} .
$$

Then,

$$
\forall i \in I_{H}^{*}: g^{* k}\left(m_{H}^{* k}\right) \leq g^{* i}\left(m_{H}^{* i}\right)
$$


Moreover, in view of the facts, $u\left(m_{L}^{* i_{0}}, g^{* i_{0}}\left(m_{L}^{* i_{0}}\right) \mid L\right) \geq u\left(m_{H}^{* k}, g^{* k}\left(m_{H}^{* k}\right) \mid L\right)$ and $u\left(m_{L}^{* i_{0}}, g^{* i_{0}}\left(m_{L}^{* i_{0}}\right) \mid H\right) \leq u\left(m_{H}^{* k}, g^{* k}\left(m_{H}^{* k}\right) \mid H\right)$, the assumption on the slopes of the two types of indifference curves implies

$$
m_{L}^{* i_{0}} \leq m_{H}^{* k} .
$$

Since each applicant tries to minimize his education level given a wage level, it follows that $\left[m_{t}^{* i}, \bar{m}\right] \in \mathcal{M}^{i}$. For two positive real numbers $\varepsilon:=$ $\left(\varepsilon_{L}, \varepsilon_{H}\right) \gg 0$, define incumbent $k$ 's strategy $g_{\varepsilon}^{k}$ by

$$
g_{\varepsilon}^{k}(m):=\left\{\begin{array}{lll}
U_{L}^{*}(0)+\varepsilon_{L} & \text { if } & 0 \leq m<m_{H}^{* k}, \\
g^{* k}\left(m_{H}^{* k}\right)+\varepsilon_{H} & \text { if } & m_{H}^{* k} \leq m .
\end{array}\right.
$$

Strategy $g_{\varepsilon}^{k}$ is $\mathcal{M}^{k}$-measurable. For any $\delta>0$ sufficiently small, we may choose $\varepsilon \ll(\delta, \delta)$ so that

$$
\begin{aligned}
u\left(0, g_{\varepsilon}^{k}(0) \mid L\right) & >u\left(m_{H}^{* k}, g_{\varepsilon}^{k}\left(m_{H}^{* k}\right) \mid L\right) \\
u\left(0, g_{\varepsilon}^{k}(0) \mid H\right) & <u\left(m_{H}^{* k}, g_{\varepsilon}^{k}\left(m_{H}^{* k}\right) \mid H\right) .
\end{aligned}
$$

Strategy $g_{\varepsilon}^{k}$ is intended to offer contract $\left(0, g_{\varepsilon}^{k}(0)\right)$ to type- $L$ applicants, and contract $\left(m_{H}^{* k}, g_{\varepsilon}^{k}\left(m_{H}^{* k}\right)\right)$ to type- $H$ applicants. The preceding two inequalities say that $g_{\varepsilon}^{k}$ is indeed incentive-compatible. In the following, we choose such $\varepsilon$.

Now, given $\left\{g^{* i}\right\}_{i \in I}$, incumbent $i$ 's gain is

$$
\pi^{* i}:=n_{L}^{* i}\left(r_{L}-g^{* i}\left(m_{L}^{* i}\right)\right)+n_{H}^{* i}\left(r_{H}-g^{* i}\left(m_{H}^{* i}\right)\right) \geq 0,
$$

where $n_{t}^{* i}:=0$ and $m_{t}^{* i}$ is arbitrary if $i \notin I_{t}^{*}$. When $k$ changes his strategy from $g^{* k}$ to $g_{\varepsilon}^{k}$, while the others keep their strategies, all the type- $L$ applicants come to $k$ to sign contract $\left(0, g_{\varepsilon}^{k}(0)\right)$ and all the type- $H$ applicants also come to $k$ to sign contract $\left(m_{H}^{* k}, g_{\varepsilon}^{k}\left(m_{H}^{* k}\right)\right)$, so $k$ 's gain becomes

$$
\begin{aligned}
\pi_{\varepsilon}^{k}:= & n_{L}\left(r_{L}-U_{L}^{*}(0)-\varepsilon_{L}\right)+n_{H}\left(r_{H}-g^{* k}\left(m_{H}^{* k}\right)-\varepsilon_{H}\right) \\
= & \left(n_{L}-n_{L}^{* i_{0}}\right)\left(r_{L}-U_{L}^{*}(0)\right)+n_{L}^{* i_{0}}\left(r_{L}-g^{* i_{0}}\left(m_{L}^{* i_{0}}\right)\right) \\
& +n_{H}\left(r_{H}-g^{* k}\left(m_{H}^{* k}\right)\right) \\
& +A_{\varepsilon}
\end{aligned}
$$

where

$$
A_{\varepsilon}:=n_{L}^{* i_{0}}\left(g^{* i_{0}}\left(m_{L}^{* i_{0}}\right)-U_{L}^{*}(0)\right)-n_{L} \varepsilon_{L}-n_{H} \varepsilon_{H} .
$$

But

$$
\begin{aligned}
& \left(n_{L}-n_{L}^{* i_{0}}\right)\left(r_{L}-U_{L}^{*}(0)\right)+n_{L}^{* i_{0}}\left(r_{L}-g^{* i_{0}}\left(m_{L}^{* i_{0}}\right)\right) \\
& +n_{H}\left(r_{H}-g^{* k}\left(m_{H}^{* k}\right)\right) \\
& \quad \geq \sum_{i \in I_{L}^{*}} n_{L}^{* i}\left(r_{L}-g^{* i}\left(m_{L}^{* i}\right)\right)+\sum_{i \in I_{H}^{*}} n_{H}^{* i}\left(r_{H}-g^{* i}\left(m_{H}^{* i}\right)\right) \\
& =\sum_{i \in I} \pi^{* i} \\
& \geq \pi^{* k} .
\end{aligned}
$$


Thus,

$$
\pi_{\varepsilon}^{k} \geq \pi^{* k}+A_{\varepsilon}
$$

For $\varepsilon$ sufficiently small, $A_{\varepsilon}>0$, so $k$ 's gain increases as he changes his strategy from $g^{* k}$ to $g_{\varepsilon}^{k}$, contradicting the definition of $\left\{g^{* i}\right\}_{i \in I}$ as an equilibrium.

(iii) If $n_{L}^{* i}>0$ and $n_{L}^{* j}>0$, then in view of (ii),

$$
u\left(0, g^{* i}(0) \mid L\right)=u\left(0, g^{* j}(0) \mid L\right)
$$

so $g^{* i}(0)=g^{* j}(0)$.

(iv) If there exists $i$ for whom $n_{H}^{* i}>0$ and $g^{* i}\left(m_{H}^{* i}\right)>r_{H}$, then for this $i$ to survive,

$$
n_{L}^{* i}>0 \text { and } g^{* i}\left(m_{L}^{* i}\right)<r_{L},
$$

which contradicts (i).

Lemma 4.2. Assume \#I $\geq 2$, and let $\left\{g^{* i}\right\}_{i \in I}$ be a feasible strategy bundle. In case (1), assume

$$
\begin{aligned}
& \forall i \in I: g^{* i}(\cdot) \leq U_{H 0}(\cdot), \\
& \exists i_{k} \in I: g^{* i_{k}}(0)=w_{0} .
\end{aligned}
$$

In subcases (2.1) and (2.2), assume

$$
\begin{aligned}
& \forall i \in I: g^{* i}(\cdot) \leq\left(U_{L, k} \bigwedge U_{H k}\right)(\cdot), \\
& \exists i_{k} \in I_{k}: g^{* i_{k}}(m)=\left\{\begin{array}{lll}
w_{L, k}, & \text { if } & m=0 \\
w_{H, k}, & \text { if } & m=m_{k} .
\end{array}\right.
\end{aligned}
$$

In subcase (2.3), assume

$$
\begin{aligned}
& \forall i \in I: g^{* i}(\cdot) \leq\left(U_{L}^{\prime *} \bigwedge U_{H l}\right)(\cdot), \\
& \exists i_{k} \in I_{k}: g^{* i_{k}}(m)=\left\{\begin{array}{lll}
\max \left\{r_{L}, w_{L}^{\prime *}\right\}, & \text { if } & m=0 \\
U_{H, l}\left(m_{k^{*}}\right), & \text { if } & m=m_{k^{*}}
\end{array}\right.
\end{aligned}
$$

Then, in cases (1) and (2), no incumbent $i \neq i_{k}$ can adopt a feasible strategy $g$ which takes applicants away from $i_{k}$, and then eventually receive a nonnegative gain.

Proof. Let $\left\{g^{* i}\right\}_{i \in I}$ be the strategy bundle and let $i_{k}$ be the incumbent given in the statement of the lemma. Suppose there exist $i \in I \backslash\left\{i_{k}\right\}$ and $i$ 's feasible strategy $g$ such that $i$ takes applicants away from $i_{k}$, and then eventually receives a nonnegative gain.

We first claim that $g$ cannot attract only type- $L$ applicants. Indeed, denote by $\left(m_{L}^{i}, g\left(m_{L}^{i}\right)\right)$ the incumbent $i$ 's offered contract that type- $L$ applicants would sign. In case (1) we have

$$
u\left(m_{L}^{i}, g\left(m_{L}^{i}\right) \mid L\right)>u\left(0, w_{0} \mid L\right)
$$


so $g\left(m_{L}^{i}\right)>w_{0}>r_{L}$. In subcases (2.1) and (2.2) we have

$$
u\left(m_{L}^{i}, g\left(m_{L}^{i}\right) \mid L\right)>u\left(0, w_{L, k} \mid L\right),
$$

so $g\left(m_{L}^{i}\right)>w_{L, k} \geq r_{L}$. In subcase (2.3) we have

$$
u\left(m_{L}^{i}, g\left(m_{L}^{i}\right) \mid L\right)>u\left(0, \max \left\{r_{L}, w_{L}^{* *}\right\} \mid L\right),
$$

so $g\left(m_{L}^{i}\right)>r_{L}$. In both cases (1) and (2), therefore, $g\left(m_{L}^{i}\right)>r_{L}$, consequently $\left(m_{L}^{i}, g\left(m_{L}^{i}\right)\right)$ yields a negative gain, and the claim was proved.

Therefore, $g$ attracts some type- $H$ applicants; let $\left(m_{H}^{i}, g\left(m_{H}^{i}\right)\right)$ be $i$ 's offered contract which is signed by type- $H$ applicants. Then,

$$
\begin{aligned}
& u\left(m_{H}^{i}, g\left(m_{H}^{i}\right) \mid H\right) \\
& > \begin{cases}u\left(0, w_{0} \mid H\right), & \text { in case }(1) ; \\
u\left(m_{k}, w_{H, k} \mid H\right), & \text { in subcases }(2.1) \text { and }(2.2) ; \\
u\left(m_{k^{*}}, U_{H, l}\left(m_{k^{*}}\right) \mid H\right), & \text { in subcase }(2.3) .\end{cases}
\end{aligned}
$$

The contract $\left(m_{H}^{i}, g\left(m_{H}^{i}\right)\right)$ then attracts all the type- $H$ applicants in both cases (1) and (2). Let $U_{L}$ be the indifference curve of the type- $L$ applicants that passes through $\left(m_{H}^{i}, g\left(m_{H}^{i}\right)\right)$.

Denote by $n_{L}^{i}$ the number of type- $L$ applicants who eventually sign a contract with $i$ after instituting $\left\{g,\left\{g^{* j}\right\}_{j \in I \backslash\{i\}}\right\}$. Then, $0 \leq n_{L}^{i} \leq n_{L}$. We claim that $n_{L}^{i}<n_{L}$. If $n_{L}^{i}=n_{L}$, then denoting by $\left(m_{L}^{i}, g\left(m_{L}^{i}\right)\right)$ the contract eventually signed by type- $L$ applicants,

$$
\begin{aligned}
u\left(m_{L}^{i}, g\left(m_{L}^{i}\right) \mid L\right) & \geq u\left(m_{H}^{i}, g\left(m_{H}^{i}\right) \mid L\right) \\
& =u\left(0, U_{L}(0) \mid L\right),
\end{aligned}
$$

so that $g\left(m_{L}^{i}\right) \geq U_{L}(0)$, and consequently

$$
\begin{aligned}
& n_{L}\left(r_{L}-g\left(m_{L}^{i}\right)\right)+n_{H}\left(r_{H}-g\left(m_{H}^{i}\right)\right) \\
& \quad \leq n_{L}\left(r_{L}-U_{L}(0)\right)+n_{H}\left(r_{H}-g\left(m_{H}^{i}\right)\right) \\
& \quad<0 .
\end{aligned}
$$

Here, the last inequality is: a consequence of $g\left(m_{H}^{i}\right)>U_{H 0}\left(m_{H}^{i}\right)$ in case (1); a consequence of $g\left(m_{H}^{i}\right)>U_{H, k}\left(m_{H}^{i}\right)$ in subcases (2.1) and (2.2); and a consequence of $g\left(m_{H}^{i}\right)>U_{H, l}\left(m_{H}^{i}\right)$ and $i \in I \backslash\left\{i_{k}\right\}$ in subcase (2.3). Thus $g$ would eventually receive a negative gain, and the claim was proved.

Due to the claim, there exists $j \neq i$ who eventually receives some type- $L$ applicants only; let $\left(m_{L}^{j}, w_{L}^{j}\right)$ be $j$ 's offered contract signed by type- $L$ applicants. For $j$ to survive, $r_{L} \geq w_{L}^{j}$. Then,

$$
\begin{aligned}
u\left(0, r_{L} \mid L\right) & \geq u\left(m_{L}^{j}, w_{L}^{j} \mid L\right) \\
& \geq u\left(m_{H}^{i}, g\left(m_{H}^{i}\right) \mid L\right) \\
& =u\left(0, U_{L}(0) \mid L\right),
\end{aligned}
$$


so that $r_{L} \geq U_{L}(0)$. Then, the three inequalities,

$$
\begin{aligned}
n_{L}\left(r_{L}-U_{L}(0)\right)+n_{H}\left(r_{H}-g\left(m_{H}^{i}\right)\right) & <0 \\
n_{L}^{i}\left(r_{L}-U_{L}(0)\right)+n_{H}\left(r_{H}-g\left(m_{H}^{i}\right)\right) & \geq 0 \\
n_{L} & \geq n_{L}^{i}
\end{aligned}
$$

are inconsistent. Thus, no incumbent $i\left(\neq i_{k}\right)$ can choose a strategy which takes applicants away from $i_{k}$ and then eventually receive a nonnegative gain.

Proof of Theorem 3.2. Let $\left\{g^{* i}\right\}_{i \in I}$ be the strategy bundle given in the statement of the theorem. Choose $i_{1} \in I$. We need to show that $i_{1}$ cannot improve upon $\left\{g^{* i}\right\}_{i \in I}$. Suppose that $i_{1}$ changes his strategy from $g^{* i_{1}}$ to $g^{i_{1}}$.

In general, if an incumbent $i$ designs a mechanism $g$ to induce type- $L$ applicants to sign contract $\left(m_{L}, g\left(m_{L}\right)\right)$ given $\left\{g,\left\{g^{* j}\right\}_{j \neq i}\right\}$, and if $m_{L}>0$, then $i$ can do better by another mechanism $g^{\prime}$ such that the type- $L$ applicants would choose contract $\left(0, g^{\prime}(0)\right)$ and $g^{\prime}(0)<g\left(m_{L}\right)$. Indeed, let $U_{L}$ be the indifference curve of the type- $L$ applicants which passes through $\left(m_{L}, g\left(m_{L}\right)\right)$. Since each applicant wants to minimize his education level given a wage level, we may assume $\left[m_{L}, \bar{m}\right] \in \mathcal{M}^{i}$. In view of the assumption on the slopes of the two types of indifference curves, if type- $H$ applicants also sign $i$ 's offered contract $\left(m_{H}, g\left(m_{H}\right)\right)$, then $\left(m_{L}, g\left(m_{L}\right)\right) \leq\left(m_{H}, g\left(m_{H}\right)\right)$. The required mechanism $g^{\prime}$ is given as

$$
g^{\prime}(m):=\left\{\begin{array}{lll}
U_{L}(0) & \text { if } & 0 \leq m<m_{L} \\
g(m) & \text { if } & m_{L} \leq m
\end{array}\right.
$$

If, on the other hand, $i$ is to induce only type- $H$ applicants with his contract $\left(m_{H}, g\left(m_{H}\right)\right)$, then again without loss of generality, $u(0, g(0) \mid L) \geq$ $u\left(m_{H}, g\left(m_{H}\right) \mid L\right)$.

Thus, we may assume without loss of generality that

$$
\forall m \in M: u\left(0, g^{i_{1}}(0) \mid L\right) \geq u\left(m, g^{i_{1}}(m) \mid L\right) .
$$

We can re-define $U_{L}$ as the indifference curve of the type- $L$ applicants that passes through $\left(0, g^{i_{1}}(0)\right)$. The above inequality means $U_{L}(\cdot) \geq g^{i_{1}}(\cdot)$.

Now, if $g^{i_{1}}(0)>w_{0}$, then $i_{1}$ attracts all the applicants of both types and the gain becomes negative, so $i_{1}$ cannot improve upon $\left\{g^{* i}\right\}_{i \in I}$.

If $g^{i_{1}}(0)<w_{0}$, then in view of $U_{L}(\cdot) \geq g^{i_{1}}(\cdot), i_{1}$ loses all type- $L$ applicants. If $i_{1}$ also loses type- $H$ applicants, he becomes inactive, so he cannot improve upon $\left\{g^{* i}\right\}_{i \in I}$. Therefore, suppose there exists $m_{H}$ for which $g^{i_{1}}\left(m_{H}\right)>$ $U_{H 0}\left(m_{H}\right)$. Then $i_{1}$ attracts all type- $H$ applicants, the other incumbents suffer a loss from type- $L$ applicants so drop out of the "market", and $i_{1}$ eventually gets all applicants. But then he cannot make a positive gain in case (1).

Suppose $g^{i_{1}}(0)=w_{0}$. If $i_{1}$ is to have a positive eventual gain, he has to attract all the type-H applicants given $\left\{g^{i_{1}},\left\{g^{* i}\right\}_{i \neq i_{1}}\right\}$. This means: 


$$
\exists m_{H} \in M: g^{i_{1}}\left(m_{H}\right)>U_{H 0}\left(m_{H}\right) .
$$

But then $i_{1}$ attracts all type- $H$ applicants, the other incumbents, as long as they keep type- $L$ applicants, suffer from a loss and drop out, so $i_{1}$ eventually gets all the applicants of both types. In case (1), $i_{1}$ 's eventual gain becomes negative.

Proof of Theorem 3.3. Let $\left\{g^{* i}\right\}_{i \in I}$ be the strategy bundle given in the statement of the theorem. Choose $i_{1} \in I_{k}$. In view of Lemma 4.2, it suffices to show that $i_{1}$ cannot improve upon $\left\{g^{* i}\right\}_{i \in I}$. Suppose that $i_{1}$ changes his strategy from $g^{* i_{1}}$ to $g^{i_{1}}$. Let $U_{L}$ be the indifference curve of the type- $L$ applicants that passes through $\left(0, g^{i_{1}}(0)\right)$. By the same argument as in the second paragraph of the proof of Theorem 3.2, we may assume without loss of generality that $U_{L}(\cdot) \geq g^{i_{1}}(\cdot)$.

If $g^{i_{1}}(0)>w_{L, k}$, then $i_{1}$ attracts all the applicants of type $L$, and the gain becomes negative (regardless whether $i_{1}$ attracts type- $H$ applicants or not) because of the definition of $k$, so $i_{1}$ cannot improve upon $\left\{g^{* i}\right\}_{i \in I}$.

If $g^{i_{1}}(0)<w_{L, k}$, then $i_{1}$ loses all type- $L$ applicants. So in order for him to stay active, $g^{i_{1}}$ has to attract all type- $H$ applicants. If $g^{i_{1}}(0)=w_{L, k}$, in order to make a change for increase in his gain, $i_{1}$ has to attract all the type- $H$ applicants. Thus, if $g^{i_{1}}(0) \leq w_{L, k}$, which we assume in the rest of the proof, $i_{1}$ has to attract all type- $H$ applicants given $\left\{g,\left\{g^{* i}\right\}_{i \neq i_{1}}\right\}$. Somebody other than $i_{1}$, say $i_{2}$, is taking strategy $g^{* i_{2}}$, which guarantees utility level $u\left(m_{k}, w_{H, k} \mid H\right)$ to the type- $H$ applicants, and utility level $u\left(0, w_{L, k} \mid L\right)$ to the type- $L$ applicants. Since $i_{1}$ has to supercede $i_{2}$ 's guarantee to the type- $H$ applicants,

$$
\exists h: I_{h} \ni i_{1} \text {, and } g^{i_{1}}\left(m_{h}\right)>U_{H, k}\left(m_{h}\right) .
$$

Incumbent $i_{2}$, and possibly some members $i \in I \backslash\left\{i_{1}, i_{2}\right\}$, receive only type- $L$ applicants (all type- $L$ applicants, in case $g^{i_{1}}(0)<w_{L, k}$ ). We consider two cases separately: (A) $r_{L}<w_{L, k}$, and (B) $r_{L}=w_{L, k}$.

Suppose (A). Then those incumbents who received type- $L$ applicants suffer from a loss and drop out of the "market". As long as $g^{i_{1}}(0)>r_{L}$, incumbent $i_{1}$ eventually gets all applicants. But then $i_{1}$ 's eventual gain becomes negative, in view of the definition of $k$. If $g^{i_{1}}(0) \leq r_{L}$, then $i_{1}$ may or may not get all type- $L$ applicants eventually, since somebody other than $i_{1}$ and $i_{2}$ may be able to keep type- $L$ applicants. In case $i_{1}$ eventually gets all type- $L$ applicants, his eventual gain is negative, in view of the definition of $k$. In case $i_{1}$ does not get all type- $L$ applicants eventually, his eventual gain is even lower than in the situation in which he gets all type- $L$ applicants, since each type- $L$ applicant brings in nonnegative gain. Thus, $i_{1}$ cannot improve upon $\left\{g^{* i}\right\}_{i \in I}$ in case (A).

Suppose (B). Then $w_{H, k}=r_{H}$, and the conditions,

$$
U_{L}(\cdot) \geq g^{i_{1}}(\cdot) \text { and } g^{i_{1}}\left(m_{h}\right)>U_{H, k}\left(m_{h}\right)
$$


mean that $i_{1}$ receives a negative gain given $\left\{g,\left\{g^{* i}\right\}_{i \neq i_{1}}\right\}$, so he cannot survive.

Proof of Theorem 3.4. Let $\left\{g^{* i}\right\}_{i \in I}$ be the strategy bundle given in the statement of the theorem. It suffices to show that $i_{k}$ cannot improve upon $\left\{g^{* i}\right\}_{i \in I}$. Literally the same proof as the proof of Theorem 3.3 (except that $i_{k}$ replaces $\left.i_{1}\right)$ applies.

Proof of Theorem 3.5. Let $\left\{g^{* i}\right\}_{i \in I}$ be the strategy bundle given in the statement of the theorem. It suffices to show that $i_{k}$ cannot improve upon $\left\{g^{* i}\right\}_{i \in I}$. Suppose $i_{k}$ changes his strategy from $g^{* i_{k}}$ to $g^{i_{k}}$. Let $U_{L}$ be the indifference curve of the type- $L$ applicants that passes through $\left(0, g^{i_{k}}(0)\right)$. Without loss of generality, $U_{L}(\cdot) \geq g^{i_{k}}(\cdot)$.

If $g^{i_{k}}(0) \geq g^{* i_{k}}(0) \geq r_{L}$, then all type- $L$ applicants stay with $i_{k}$, contributing a nonpositive gain, so $i_{k}$ has to keep all type- $H$ applicants. Then $i_{k}$ 's eventual gain is no higher than that before his change of strategy, in view of the definition of $k^{*}$. So $i_{k}$ cannot improve upon $\left\{g^{* i}\right\}_{i \in I}$.

If $g^{i_{k}}(0)<g^{* i_{k}}(0)$, then $i_{k}$ loses all type- $L$ applicants, so in order to stay active, the mechanism $g^{i_{k}}$ is designed so that it keeps all type- $H$ applicants given $\left\{g^{i_{k}},\left\{g^{* i}\right\}_{i \neq i_{k}}\right\}$. We consider two cases separately: (A) $r_{L}<w_{L}^{\prime *}$ (so that $\left.g^{* i_{k}}(0)=w_{L}^{\prime *}>r_{L}\right)$, and (B) $r_{L} \geq w_{L}^{\prime *}$ (so that $g^{* i_{k}}(0)=r_{L}$ ).

Suppose (A). Then incumbent $i_{p}$, and possibly some members $i \in I \backslash$ $\left\{i_{k}, i_{p}\right\}$, get all the type- $L$ applicants, who bring in only a loss, so those incumbents who receive type- $L$ applicants eventually drop.

If $g^{i_{k}}(0)>r_{L}$, incumbent $i_{k}$ eventually takes back all the type- $L$ applicants. But then $i_{k}$ 's eventual gain becomes no greater than his original gain given $\left\{g^{* i}\right\}_{i \in I}$, in view of the definition of $k^{*}$.

If $g^{i_{k}}(0)<r_{L}$, then incumbent $i^{\prime}$ eventually gets all the type- $L$ applicants. Incumbent $i_{k}$ eventually ends up only with the type- $H$ applicants with a contract $\left(m_{h}, g^{i_{k}}\left(m_{h}\right)\right)$ for some $h \in J_{i_{k}}$. But then $i_{k}$ 's eventual gain becomes:

$$
\begin{aligned}
n_{H} & \left(r_{H}-g^{i_{k}}\left(m_{h}\right)\right) \\
& \leq n_{H}\left(r_{H}-U_{H, l}\left(m_{h}\right)\right) \\
& =n_{L}\left(r_{L}-\max \left\{r_{L}, g^{i_{k}}(0)\right\}\right)+n_{H}\left(r_{H}-U_{H, l}\left(m_{h}\right)\right) \\
& \leq\left(r_{L}-\max \left\{r_{L}, w_{L}^{\prime *}\right\}\right)+n_{H}\left(r_{H L}-U_{H, l}\left(m_{k^{*}}\right)\right),
\end{aligned}
$$

so $i_{k}$ cannot improve upon $\left\{g^{* i}\right\}_{i \in I}$.

If $g^{i_{k}}(0)=r_{L}$, incumbent $i_{k}$ may get back some of the type- $L$ applicants, but they bring in only zero gain, so the above inequalities apply here as well; $i_{k}$ 's eventual gain becomes no greater than his original gain given $\left\{g^{* i}\right\}_{i \in I}$.

Suppose (B). Then, $i_{k}$, if he survives, eventually ends up only with the type- $H$ applicants. But $i_{k}$ 's gain given $\left\{g^{* i}\right\}_{i \in I}$ comes only from the type- $H$ applicants, so as in the preceding two paragraphs, $i_{k}$ cannot improve upon $\left\{g^{* i}\right\}_{i \in I}$. 
Sketch of Proof of Theorem 3.7. Let $\left\{g^{* i}\right\}_{i \in I}$ be the strategy bundle given in the theorem. For subcase (3.1), choose any $i_{k} \in I$; for subcase (3.2.1), set $i_{k}:=i_{1}$; for the other subcases, $i_{k}$ is already defined.

We first establish that no incumbent $i \neq i_{k}$ can adopt a feasible strategy $g$ which takes applicants away from $i_{k}$, and eventually receive a nonnegative gain. The proof follows closely the proof of Lemma 4.2. Here is an outline: Suppose there exist an incumbent $i \neq i_{k}$ and his feasible strategy $g$ such that $i$ takes applicants away from $i_{k}$, and eventually receives a nonnegative gain. As in the proof of Lemma 4.2, we claim that $i$ cannot attract only type- $L$ applicants, so $g$ attracts all type- $H$ applicants, that is, there exists $m_{H}^{i} \in M$ such that

$$
\begin{aligned}
& u\left(m_{H}^{i}, g\left(m_{H}^{i}\right) \mid H\right) \\
& \quad> \begin{cases}u\left(0, w_{0} \mid H\right), & \text { in subcase (3.1); } \\
u\left(m_{k}, \underline{w}_{H, k} \mid H\right), & \text { in subcases (3.2.1)-(3.2.2); } \\
u\left(m_{k^{*}}, \underline{U}_{H, l}\left(m_{k^{*}}\right) \mid H\right), & \text { in subcase (3.2.3). }\end{cases}
\end{aligned}
$$

Without loss of generality, $\left[m_{H}^{i}, \bar{m}\right] \in \mathcal{M}^{i}$. By definition of $k$ and by the fact that $i \neq i_{k}$, either (A) $g\left(m_{H}^{i}\right)>r_{H}$ or else (B) $n_{L}\left(r_{L}-U_{L}(0)\right)+$ $n_{H}\left(r_{H}-g\left(m_{H}^{i}\right)\right)<0$. If $(\mathrm{A})$ is the case, $i$ receives a loss from type- $H$ applicants, so he has to attract type- $L$ applicants also. But the only way to attract type- $L$ applicants is to offer a wage higher than $r_{L}$ (thereby receiving a loss also from type- $L$ applicants), in view of $g^{* i_{k}}(0) \geq r_{L}$. So, $i$ cannot survive. If (B) is the case, the same argument as in the proof of Lemma 4.2 applies.

We only need to show that $i_{k}$ cannot improve upon $\left\{g^{* i}\right\}_{i \in I}$. Suppose $i_{k}$ changes his strategy from $g^{* i_{k}}$ to $g^{i_{k}}$. Let $U_{L}$ be the indifference curve of the type- $L$ applicants that passes through $\left(0, g^{i_{k}}(0)\right)$. Without loss of generality, $U_{L}(\cdot) \geq g^{i_{k}}(\cdot)$.

Consider subcase (3.1). If $g^{i_{k}}(0)>w_{0}$, then the proof of Theorem 3.2 applies. If $g^{i_{k}}(0) \leq w_{0}$, then, as in the proof of Theorem 3.2, there exists $m_{H} \in M$ such that $g^{i_{k}}\left(m_{H}\right)>U_{H 0}\left(m_{H}\right)$. In subcase (3.1), this means either (A) $g^{i_{k}}\left(m_{H}\right)>r_{H}$, or else (B) $n_{L}\left(r_{L}-g^{i_{k}}(0)\right)+n_{H}\left(r_{H}-g^{i_{k}}\left(m_{H}\right)\right)<0$. If (A) is the case, $i_{k}$ receives a loss from type- $H$ applicants. In order to survive, therefore, $i_{k}$ has to attract type- $L$ applicants with wage $g^{i_{k}}(0)$ lower than $r_{L}$, but this is impossible in view of the fact that $g^{* i}(0)=w_{0} \geq r_{L}$ for all $i \neq i_{k}$. If (B) is the case, the proof of Theorem 3.2 applies.

The idea of the proofs of the theorem for subcases (3.2.1)-(3.2.3) are the same as above: We follow the proofs of Theorems 3.3-3.5. The only situation in which we have to modify the proof is the case $g^{i_{k}}\left(m_{H}\right)>r_{H}$ (in which case, it is possible that $\left.n_{L}\left(r_{L}-g^{i_{k}}(0)\right)+n_{H}\left(r_{H}-g^{i_{k}}\left(m_{H}\right)\right) \geq 0\right)$. But in this situation $i_{k}$ receives a loss from type- $H$ applicants, so in order for him to receive a nonnegative eventual gain, he has to attract type- $L$ applicants with lower wage than $r_{L}$, which is impossible in the presence of $i^{\prime}$.

Proof of Proposition 3.8. Suppose that there exists a noncooperative equilibrium $\left\{g^{i}\right\}_{i \in I}$ for which 


$$
\exists i^{*} \in I: \exists m^{*} \in M: g^{i^{*}}\left(m^{*}\right)>\left(U_{H 0} \bigvee U_{H, k}\right)\left(m^{*}\right)
$$

Let $\left\{\left(n_{L}^{i}, n_{H}^{i}\right)\right\}_{i \in I}$ be the associated assignment, and let $I_{t}$ be the set of incumbents $i$ whose contract $\left(m_{t}^{i}, g^{i}\left(m_{t}^{i}\right)\right)$ is actually signed by some applicants of type $t \in\{L, H\}$. By Lemma $4.1, g^{i}\left(m_{H}^{i}\right) \leq r_{H}$ for all $i \in I_{H}, m_{L}^{i}=0$ for all $i \in I_{L}$, and $w_{L}:=g^{i}(0)=g^{j}(0)$ for all $i, j \in I_{L}$.

For $i \in I_{H}$, type- $H$ applicants sign the contract $\left(m_{H}^{i}, g^{i}\left(m_{H}^{i}\right)\right)$, when they could sign the contract $\left(m^{*}, g^{i^{*}}\left(m^{*}\right)\right)$, so

$$
\forall i \in I_{H}: u\left(m_{H}^{i}, g^{i}\left(m_{H}^{i}\right) \mid H\right) \geq u\left(m^{*}, g^{i^{*}}\left(m^{*}\right) \mid H\right)
$$

in short, each contract $\left(m_{H}^{i}, g^{i}\left(m_{H}^{i}\right)\right)$ is strictly above the indifference curves $U_{H, k}$ and $U_{H 0}$, for all $i \in I_{H}$. For each $i \in I_{H}, m_{H}^{i} \in\left\{0, m_{1}, m_{2}, \cdots\right\}$, since every applicant minimizes the needed education level. Define $w_{L}^{i} \in \mathbf{R}_{+}$by

$$
u\left(0, w_{L}^{i} \mid L\right)=u\left(m_{H}^{i}, g^{i}\left(m_{H}^{i}\right) \mid L\right) .
$$

Choose any $j \in I_{L}$. Since type- $L$ applicants sign the contract $\left(0, w_{L}\right)$ rather than the contract $\left(m_{H}^{i}, g^{i}\left(m_{H}^{i}\right)\right)$ for any $i \in I_{H}$,

$$
\begin{aligned}
u\left(0, w_{L} \mid L\right) & \geq u\left(m_{H}^{i}, g^{i}\left(m_{H}^{i}\right) \mid L\right) \\
& =u\left(0, w_{L}^{i} \mid L\right)
\end{aligned}
$$

consequently,

$$
w_{L} \geq w_{L}^{\prime i}
$$

Therefore,

$$
\begin{aligned}
\sum_{j \in I_{L}} n_{L}^{j}\left(r_{L}-w_{L}\right) & =\sum_{i \in I_{H}} \frac{n_{H}^{i}}{n_{H}} n_{L}\left(r_{L}-w_{L}\right) \\
& \leq \sum_{i \in I_{H}} \frac{n_{H}^{i}}{n_{H}} n_{L}\left(r_{L}-w_{L}^{i}\right)
\end{aligned}
$$

In view of the present assumption, $g^{i}\left(m_{H}^{i}\right)>\max \left\{U_{H, k}\left(m_{H}^{i}\right), U_{H 0}\left(m_{H}^{i}\right)\right\}$ for all $i \in I_{H}$, and the definition of $k$,

$$
\begin{aligned}
& \sum_{j \in I_{L}} n_{L}^{j}\left(r_{L}-w_{L}\right)+\sum_{i \in I_{H}} n_{H}^{i}\left(r_{H}-g^{i}\left(m_{H}^{i}\right)\right) \\
& \quad \leq \sum_{i \in I_{H}} \frac{n_{H}^{i}}{n_{H}}\left[n_{L}\left(r_{L}-w_{L}^{i}\right)+n_{H}\left(r_{H}-g^{i}\left(m_{H}^{i}\right)\right)\right] \\
& \quad<0 .
\end{aligned}
$$

Thus, some active incumbents suffer from a loss, contradicting the definition of $\left\{g^{i}\right\}_{i \in I}$ as a noncooperative equilibrium. 


\section{References}

Ichiishi, T.: Microeconomic Theory. Oxford, U.K.: Blackwell Publishers 1997

Ichiishi, T., Idzik A.: Bayesian cooperative choice of strategies. International Journal of Game Theory 25, 455-473 (1996)

Ichiishi, T., Idzik A., Zhao J.: Cooperative processing of information via choice at an information set. International Journal of Game Theory 23, 145-165 (1994)

Ichiishi, T., Radner R.: A profit-center game with incomplete information. 1997 (forthcoming in Review of Economic Design)

Ichiishi, T., Sertel M.: Cooperative interim contract and re-contract: Chandler's M-form firm. Economic Theory 11, 523-543 (1998)

Spence, M.: Competitive optimal responses to signals: an analysis of efficiency and distribution. Journal of Economic Theory 7, 296-332 (1974)

Vohra, R.: Incomplete information, incentive compatibility and the core. Journal of Economic Theory 86, 123-147 (1999)

Wilson, R.: Information efficiency, and the core of an economy. Econometrica 46, 807-816 (1978)

Yannelis, N.C.: The core of an economy with differential information. Economic Theory 1, 183-198 (1991) 